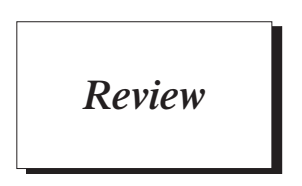

\title{
Thoracic Endovascular Aortic Repair-indications and Evidence
}

\author{
Christopher Q. Cao, BSc (Med), MBBS, ${ }^{1,2}$ Paul G. Bannon, MBBS, PhD, FRACS, ${ }^{1,2}$ \\ Rachel Shee, MBBS, ${ }^{1,2}$ and Tristan D. Yan, BSc (Med), MBBS, PhD ${ }^{1,2}$
}

\begin{abstract}
Purpose: Since its introduction more than a decade ago, thoracic endovascular aortic repair (TEVAR) has shown promising results for patients with various thoracic aortic diseases. The aim of the current review is to assess the current literature to evaluate the safety and efficacy of TEVAR.

Methods: A thorough search of the existing literature on TEVAR was conducted on electronic databases, including Medline, Pubmed, EMBASE and Database of Abstracts of Review of Effectiveness. The most recent results were categorized according to the indications of performing TEVAR.

Results: A number of case-series studies and reviews have shown reduced early morbidity and mortality rates in a range of thoracic aortic diseases for TEVAR in comparison to open surgical repair. However, there is a lack of robust clinical data to suggest any improvement in long-term overall survival.

Conclusion: Despite numerous encouraging results from a large number of publications in recent years, there remains a lack of level 1 evidence to support an improvement of long-term overall survival for patients who underwent TEVAR when compared with traditional treatment modalities. There appears to be an urgent need to conduct well-designed randomized-controlled trials in this rapidly expanding intervention.
\end{abstract}

Key words: thoracic endovascular aortic repair, aortic dissection, aortic aneurysm

\section{Introduction}

Despite recent improvements in cardiothoracic surgery, open surgical repair for thoracic aortic disease is still

${ }^{1}$ Department of Cardiothoracic Surgery, The University of Sydney, The Royal Prince Alfred Hospital, Sydney, Australia

${ }^{2}$ The Baird Institute for Applied Heart and Lung Surgical Research, Sydney, Australia

Received: August 4, 2010; Accepted: December 6, 2010

Corresponding author: Tristan D. Yan, BSc (Med), MBBS, PhD.

The University of Sydney, Department of Cardiothoracic Surgery, Royal Prince Alfred Hospital, Missenden Road, Camperdown NSW 2050, Sydney, Australia

Email: Tristan.Yan@hotmail.com

(C)2011 The Editorial Committee of Annals of Thoracic and Cardiovascular Surgery. All rights reserved. associated with significant morbidity and mortality. In the current literature, the peri-operative mortality rate after open repair for aortic aneurysms is reported to be approximately $10 \%-20 \%$ and significant morbidity ranges from $30 \%-50 \%{ }^{1,2)}$ It has been anticipated that the introduction of thoracic endovascular aortic repair (TEVAR) will improve the peri-procedural and long-term outcomes of thoracic aortic disease. In recent years, the indications involving the off-label use of TEVAR have expanded to include descending thoracic aortic aneurysms, dissections, penetrative aortic ulcers and traumatic aortic injuries. Despite encouraging results, there remains a lack of robust clinical data on long-term outcomes in this fast evolving technology. This review will focus on the current clinical outcomes of TEVAR in patients with thoracic aortic pathology. 


\section{Thoracic Aortic Aneurysm}

\section{Natural History}

The linear relationship between the growth rate and the size of aortic aneurysms has been long recognized. For an aneurysm of $4 \mathrm{~cm}$ in diameter, the growth rate is approximately $0.1 \mathrm{~cm}$ per year, compared to an $8 \mathrm{~cm}$ aneurysm, which can have a growth rate of up to $0.2 \mathrm{~cm}$ per year.") Davies et al. reported that patients with aneurysms larger than $6 \mathrm{~cm}$ had an annual risk of death, rupture, or dissection of $15.6 \% .^{4)}$ Based on these historical data, the conservative criteria for surgical intervention were set at $5.5 \mathrm{~cm}$ for ascending and $6.5 \mathrm{~cm}$ for descending aneurysms. The current evidence does not suggest that aneurysms of less than $5.5 \mathrm{~cm}$ in diameter benefit from surgical repair except in patients with connective tissue disease, strong family history or symptomatic disease. ${ }^{5)}$

\section{Gore TAG Thoracic Endograft}

The Gore TAG device was the first commercially available stent-graft product to be tested in clinical trials, in the United States. Initial studies were halted in November 2001 after the discovery of longitudinal spine fractures in the stent. Since then, modifications have been made to the device, including the elimination of the longitudinal wire and incorporation of a new polytetrafluoroethylene material. Makaroun et al. conducted a prospective phase II study in 17 USA centres, of the Gore TAG endograft in 142 patients with descending thoracic aneurysms. ${ }^{6}$ Patients were excluded if they had aneurysmal rupture or dissection. The mean follow-up of this study was 24 months and routine imaging was reported to be obtained at regular intervals. The authors of this study highlighted that $90 \%$ of patients included in their trial were American Society of Anaesthesiologist category III or IV. After excluding three patients who had unsuccessful deployment, the overall and aneurysm-related mortality rates at 2 years were reported to be $25 \%$ and $3 \%$, respectively. Overall, major complications occurred in 45 patients (32\%) within 30 days of surgery, with the most common complications being local vascular complications, cardiopulmonary events and bleeding. The operative mortality rate was $2 \%$. During follow-up, 20 fractures were identified in 19 patients over a 2-year period, with only one patient developing a clinical event and requiring treatment as a result of a type III endoleak.

Bavaria et al. compared the results of the multi-institutional study with a nonrandomized group of patients $(n=94)$ who underwent open surgical repair from the same institu- tions; patients who underwent TEVAR had a significant reduction in perioperative mortality (2.1\% vs $11.7 \%)$, respiratory failure (4\% vs 20\%), renal insufficiency ( $1 \%$ vs $13 \%$ ), spinal cord ischemia (3\% vs $14 \%$ ), mean length of ICU stay ( $2.6 \pm 14.6$ vs $5.2 \pm 7.2$ days) and overall hospital stay $\left(7.4 \pm 17.7\right.$ vs $14.4 \pm 12.8$ days). ${ }^{7}$ However, after 2 years follow-up, three patients from the TEVAR group required reintervention whilst none was required for patients in the open group, and there was no difference in overall mortality between the two groups. In a more recent comparative study using the same cohort of patients at five years follow-up, aneurysm-related mortality was again found to be lower for TAG patients $(2.8 \%$ vs $11.7 \%)$; however, all-cause mortality was not significantly different (68\% vs 67\%) between the two groups. ${ }^{8)}$ It should be noted that $26 \%$ of TAG patients and $33 \%$ of open surgery patients did not complete the 5-year follow-up, and autopsies were rarely performed.

\section{Medtronic Talent Thoracic Stent Graft}

Fattori et al. published data collected over an 8-year period, from 457 patients in seven European centres who underwent TEVAR with the Medtronic Talent device.") Although a significant proportion of these patients presented with atherosclerotic (30\%) or posttraumatic aneurysms (18\%), it should be noted that the most common indication for TEVAR in this study was aortic dissection (39.4\%). Amongst 422 patients who survived the interventional procedure, mortality during follow-up was $8.5 \%$. However, the follow-up period was variable $(24 \pm 19.4$ months, range 1-85.1 months) and imaging follow-up was available for less than $21 \%$ of patients after 3 years. Of the deceased patients, 11 deaths $(2.6 \%)$ were directly related to their aortic disease. Of these, seven patients had persistent endoleaks with aortic rupture. Overall survival at 1-, 3- and 5-years were $91 \%, 85 \%$ and $77 \%$, respectively. Furthermore, freedom from a second procedure was reported to be $92 \%, 81 \%$ and $70 \%$ over the same intervals. Technical failure, including failure to complete stent-graft deployment, was reported in 10 patients. Three patients required an immediate conversion to open repair. In-hospital complications occurred in $12.7 \%$ of patients, with stroke, local vascular complications and paraplegia being the most common. The authors of this study concluded that the endovascular treatment for thoracic aortic disease with the Talent stent was associated with low early morbidity and mortality rates, as well as adequate durability as indicated by a high freedom interval from secondary intervention. However, they acknowledged that their retrospective 
study was limited by variable inclusion criteria and the absence of autopsy reports could have underestimated aortic rupture as a possible cause of death.

\section{Zenith TX2 Endovascular Graft}

Matsumura et al. reported an international nonrandomized controlled clinical trial of TEVAR using the Zenith TX2 endovascular graft $(n=130)$ versus open surgery $(\mathrm{n}=70)$ for patients with descending thoracic aortic aneurysms and large ulcers. ${ }^{10)}$ In both groups, aneurysms accounted for the majority of presentations ( $86 \%$ and $90 \%$, respectively). Forty-one percent of patients from the TEVAR group were classified as ASA category III or higher. Results of this study found the 30-day survival to be noninferior for the TEVAR group ( $98.1 \%$ vs $94.3 \%, \mathrm{p}<0.01)$. In addition, the severe morbidity composite index $(0.2 \pm 0.7$ vs $0.7 \pm 1.2)$ and the cumulative major morbidity scores $(1.3 \pm 3.0$ vs $2.9 \pm 3.6)$ at 30 days were both significantly lower in the TEVAR group. However, at 1-year follow-up, overall mortality ( $8.4 \%$ vs $14.5 \%)$ and aneurysm-related mortality (5.8\% vs $11.8 \%)$ between the two groups were not significantly different. In addition, at 1-year follow-up after TEVAR, patients were found to have progressive aneurysmal growth in $7.1 \%$, endoleak in $3.9 \%$, and migration in $2.8 \%$. Reintervention rate was reported to be similar between the two groups (4.4\% vs $5.7 \%$ ). The authors concluded that TEVAR with the TX2 device is a safe and effective alternative to open surgical repair for the treatment of anatomically suitable descending aortic aneurysms and ulcers.

\section{Ruptured Descending Thoracic Aneurysms}

Differences in aneurysmal pathology at the time of presentation may account for some variations in procedural outcomes between different institutions after TEVAR. For patients who present with ruptured descending thoracic aneurysms, the most comprehensive data have been collated by Jonker et al. ${ }^{11}$, who recently published a metaanalysis comparing open versus endovascular techniques for this subgroup of patients. Extracting data from 24 studies involving 143 patients treated by TEVAR and 81 patients with open surgery, the authors found a significantly lower 30-day mortality rate in the TEVAR group (OR, 2.15; $\mathrm{p}=0.016$ ). However, long-term follow up revealed 5 aneurysm-related deaths in the TEVAR group after 30-days, whilst no patients died of aneurysm-related causes in the open group after the same period. This comparison was limited in that $83 \%$ of patients in the open surgery group was lost during follow-up.

\section{Thoracic Aortic Dissection}

Thoracic aortic dissection originates from an intimal tear that creates a false channel in the aortic media. Blood within the false channel can propagate in either an antegrade or a retrograde fashion. The Stanford system (type A versus type B) or the De Bakey system (type 1,2 and 3) is used to categorize the aortic dissection, according to the extent of disease. ${ }^{12)}$ Stanford type A dissection involves the ascending thoracic aorta, which can cause malperfusion to the brain, obliteration of the coronary arterial flow or cardiac tamponade. Both type A and type B diseases can cause malperfusion of the spinal cord, bowel, liver, kidneys and the lower limbs. ${ }^{13)}$

\section{Type A Aortic Dissection}

Currently, open surgical intervention remains to be the standard procedure for managing type A dissections. ${ }^{12)}$ Results from the International Registry of Acute Aortic Dissection (IRAD) study reported that the in-hospital mortality rate for surgical intervention in this group of patients was $26 \%$, compared to $58 \%$ in patients who were treated by medical therapy. However, selection bias was likely to be present as patients with worse baseline features were excluded from surgical intervention. ${ }^{14)}$ The clinical experience of using TEVAR in type A aortic dissection is limited to a few case reports, and it has been mainly used on a compassion basis.

\section{Type B Aortic Dissection}

Thoracic type B dissection does not involve the ascending aortic segment of the thoracic aorta. Management of type B disease can be categorized into type B dissection without complications and type $\mathrm{B}$ dissection with complications, but most studies include a combination of both. The current clinical evidence for utilizing TEVAR for type B dissection originates from an initial report by Dake and colleagues in 1999. ${ }^{15)}$ This study examined 19 patients who underwent TEVAR for type A $(n=4)$ or type $B(n=15)$ dissection. The authors reported a $100 \%$ technical success rate for stent placement, with complete thrombosis at the thoracic aortic false lumen being achieved in $79 \%$ of patients and partial thrombosis in the remaining $21 \%$. The 30 -day mortality rate was $16 \%$, with no deaths or incidence of aneurysm rupture during the subsequent follow-up period of 13 months. These encouraging results were followed by a number of larger retrospective case-series and comparative studies. ${ }^{16,17)}$ 


\section{Type B Aortic Dissection with Complications}

In a recent systematic review, Parker and Golledge reported the findings of multiple centres over 10 years, of 942 patients who underwent TEVAR for acute type B dissection with complications. ${ }^{18)}$ Only centres with 10 or more patients were including in the study, which may have resulted in selection bias. From these selected reports, procedural success was achieved in $95 \%$ of all cases, with emergency conversion being required in only $0.6 \%$ of patients. Overall in-hospital mortality was $9.1 \%$, with an early complication rate of $8.1 \%$. After an average follow-up of 20 months, re-intervention was required in $10.4 \%$ of patients, with aortic rupture in $0.8 \%$ of the cases. Average overall survival was $88 \%$ at 20 months. The authors of this review believe TEVAR offers favourable short-term outcomes and is a feasible, alternative treatment option for acute type B dissections, especially in experienced centres. This reiterates previous findings by Eggebrecht et al., who found that centres who had treated more than 20 patients have a significantly higher procedural success rate, lower overall and neurological complications and a lower 30-day mortality rate than centres that had treated less than 20 patients. ${ }^{19}$

\section{Type B Aortic Dissection without Complications}

In acute type $\mathrm{B}$ dissection without complications, antiimpulsive medical therapy is traditionally used to obtain strict blood pressure control and to minimize the risk for further complications such as rupture, malperfusion and hemodynamic instability. ${ }^{5,14)}$ Since uncomplicated type B disease is more benign than type A and complicated type $\mathrm{B}$ diseases, conservative medical management of uncomplicated type $\mathrm{B}$ dissection constitutes a benchmark that endovascular treatment is currently unable to match. ${ }^{5)}$

To demonstrate the efficacy of TEVAR in patients with uncomplicated type B aortic dissections, Nienaber and colleagues published the first randomized-controlled trial in $2009 .{ }^{20)} \mathrm{A}$ total of 140 patients with stable type B dissections were selected and randomly assigned into an elective TEVAR plus medical therapy group $(n=72)$ or a medical therapy alone group $(n=68)$. The device used in this study was the TALENT stent graft (Medtronic, Inc, Santa Rosa) and the authors acknowledged sponsorship and external monitoring from the Medtronic Bakken Research Institute. The primary end point of the study was survival at 2 years, with secondary end points examining aorta-related death, progressive aortic pathology, and morphological evidence of aortic remodelling. In the TEVAR group, the procedural success rate was reported as $95.7 \%$, with no intraoperative deaths or conversions to open surgery. The 30-day mortality rate of this group was $2.8 \%$. At the end of the trial, results of this study found no significant difference in overall survival at 2 years for patients who had optimal medical therapy compared to TEVAR and medical therapy $(95.6 \pm 2.5 \%$ vs $88.9 \pm 3.7 \%, p=0.15)$. In regards to aortic remodelling, morphological evaluation found significantly more truelumen expansion, false-lumen shrinkage and false-lumen thrombosis in the thoracic aorta for patients who had TEVAR. Ultimately, the authors of this study concluded that the trial was underpowered. However, they proposed that deferred endovascular therapy is feasible and safe for patients who fail to respond to medical management. From the available data, it was difficult to see the direct translation of improved aortic remodelling to improved long term survival. In addition, modern optimal medical treatment provided better than expected outcomes and the results of this study verified that not all patients with uncomplicated type B dissections are indicated for endovascular intervention as a first-line treatment.

\section{Penetrating Aortic Ulcer}

Although the incidences of penetrating aortic ulcer (PAU) and intramural haematoma (IMH) have increased in recent years from improved high resolution imaging, their natural history and pathophysiology remain largely unknown. ${ }^{21}{ }^{22)}$ The PAU was originally described as 'an atherosclerotic lesion with ulceration that penetrates the internal elastic lamina', not unlike a peptic ulcer on imaging. ${ }^{23)}$ In comparison, IMH is a bleeding within the walls of the aorta without an intimal tear, presumably as a result of rupture from the vaso vasora. ${ }^{22)}$ Despite being categorized as two separate classes in the Svensson system, ${ }^{12)}$ these two entities both belong to the 'acute aortic syndrome' and both can progress to aortic dissection or rupture. Similar to aortic dissection, the threshold to consider TEVAR in patients with IMH has been lowered for for those with disease in the ascending aorta or in those who are symptomatic despite medical treatment. ${ }^{22)}$ Eggebrecht et al. comprehensively reviewed the current literature on PAU, and analysed the data of 209 of these patients who underwent TEVAR. ${ }^{21)}$ Technical failure was reported to be $2 \%$, with complete sealing of PAU achieved in $96 \%$ of patients. The overall in-hospital mortality was $7 \%$, with an additional $2 \%$ aorta-related mortality rate during the mean follow-up period of 14.3 months. Although there is no agreed first-line treatment for incidental PAU, 
the authors of this study concluded that TEVAR should be indicated in symptomatic patients complicated by pseudoaneurysm formation or rupture.

\section{Traumatic Aortic Injury}

Traumatic aortic injuries (TAI) have long been recognized as having a dismal prognosis, with a mortality rate of more than $90 \% .^{24)}$ In recent years, a number of caseseries reports have demonstrated encouraging results in the short- and medium-term follow-up periods for patients who underwent TEVAR after TAI. ${ }^{25,26)} \mathrm{A}$ recent meta-analysis of data from 17 studies published between 2003 and 2007 identified 369 patients who underwent open repair and 220 who underwent TEVAR for traumatic descending thoracic aortic rupture. ${ }^{27)}$ All studies were non-randomized retrospective cohort studies, and there was limited data on baseline characteristics of the two groups. From the available data, the injury severity score was reported to be significantly higher in patients who underwent TEVAR than open repair. Despite this, procedure-related and 30-day overall mortality rates were both significantly lower in the TEVAR group than in the open repair group. In addition, of the studies that reported on postoperative paraplegia. ${ }^{27)}$ overall complication rates were significantly lower for patients who underwent endoluminal repair. A number of challenges identified by previous reports on TEVAR for patients with TAI relate to their younger age group. Specifically, collapse of the stent graft due to the acute angle of the arch and limited size of the femoral arteries causing injuries at the access site has been described. ${ }^{25,27)}$ More recently, Jonker et al. conducted a large retrospective analysis using data from the New York Statewide Planning and Research Cooperative System. ${ }^{28)}$ In this database, 328 patients were found to have undergone open surgery $(79.6 \%)$ or TEVAR $(20.4 \%)$ for traumatic thoracic aortic injury between 2000 and 2007. Although there were more major injuries for patients in the TEVAR group, the authors reported a significantly lower mortality rate $(6.0 \%$ vs $16.9 \%, \mathrm{p}=0.024)$ and fewer pulmonary complications ( $23.9 \%$ vs $37.9 \%$ ) when compared to the open surgery group. Despite these positive findings, this study also highlighted several device-related complications, such as endoleak and distal embolization, which were each identified in $9 \%$ of patients who underwent TEVAR.

\section{Summary}

Since the landmark report by Dake et al. in 1999, there has been a heightened interest in the application of thoracic endovascular aortic repair for a range of aortic diseases, including descending thoracic dissections, aneurysms, penetrating aortic ulcers, intramural haemorrhages, and traumatic aortic injuries. Despite a large number of retrospective studies demonstrating encouraging short-term results in both mortality and morbidity outcomes, there is currently no level la or $1 \mathrm{~b}$ evidence to suggest significantly improved long-term overall survival in any of these conditions. In addition, the follow-up for most studies has been relatively short, with the majority of studies reporting less than 2 years of follow-up. Recognized peri-procedural complications of TEVAR include cardiopulmonary events, cerebrovascular accidents, local vascular injury and paraplegia. Longer-term complications include persistent endoleak and the need for endovascular reintervention or conversion to open operation. In view of the increasing utilization of this relatively novel technology, there is an urgent need to conduct further studies to examine the long-term safety and efficacy of TEVAR, ideally in the form of well-designed randomized-controlled trials compared with open surgery and conservative medical management.

\section{Disclosure Statement}

Authors declare no potential conflicts of interest.

\section{References}

1) Schermerhorn ML, Giles KA, Hamdan AD, Dalhberg SE, Hagberg R, et al. Population-based outcomes of open descending thoracic aortic aneurysm repair. J Vasc Surg 2008; 48: 821-7.

2) Estrera AL, Rubenstein FS, Miller CC 3rd, Huynh TT, Letsou GV, et al. Descending thoracic aortic aneurysm: surgical approach and treatment using the adjuncts cerebrospinal fluid drainage and distal aortic perfusion. Ann Thorac Surg 2001; 72: 481-6.

3) Coady MA, Rizzo JA, Hammond GL, Mandapati D, Darr U, et al. What is the appropriate size criterion for resection of thoracic aortic aneurysms? J Thorac Cardiovasc Surg 1997; 113: 476-91.

4) Davies RR, Goldstein LJ, Coady MA, Tittle SL, Rizzo $\mathrm{JA}$, et al. Yearly rupture or dissection rates for thoracic aortic aneurysms: simple prediction based on size. Ann Thorac Surg 2002; 73: 17-27.

5) Svensson LG, Kouchoukos NT, Miller DC, Bavaria JE, Coselli JS, et al. Expert consensus document on the treatment of descending thoracic aortic disease 
using endovascular stent-grafts. Ann Thorac Surg; 85 Suppl: S1-41.

6) Makaroun MS, Dillavou ED, Kee ST, Sicard G, Chaikof E, et al. Endovascular treatment of thoracic aortic aneurysms: results of the phase II multicenter trial of the GORE TAG thoracic endoprosthesis. J Vasc Surg 2005; 41: 1-9.

7) Bavaria JE, Appoo JJ, Makaroun MS, Verter J, Yu ZF, et al. Endovascular stent grafting versus open surgical repair of descending thoracic aortic aneurysms in low-risk patients: a multicenter comparative trial. J Thorac Cardiovasc Surg 2007; 133: 369-77.

8) Makaroun MS, Dillavou ED, Wheatley GH, Cambria RP, Gore TAG Investigators. Five-year results of endovascular treatment with the Gore TAG device compared with open repair of thoracic aortic aneurysms. J Vasc Surg 2008; 47: 912-8.

9) Fattori R, Nienaber CA, Rousseau H, Beregi JP, Heijmen $\mathrm{R}$, et al. Results of endovascular repair of the thoracic aorta with the Talent Thoracic stent graft: the Talent Thoracic Retrospective Registry. J Thorac Cardiovasc Surg 2006; 132: 332-9.

10) Matsumura JS, Cambria RP, Dake MD, Moore RD, Svensson LG, et al. International controlled clinical trial of thoracic endovascular aneurysm repair with the Zenith TX2 endovascular graft: 1-year results. J Vasc Surg 2008; 47: 247-57.

11) Jonker FH, Trimarchi S, Verhagen HJ, Moll FL, Sumpio $\mathrm{BE}$, et al. Meta-analysis of open versus endovascular repair for ruptured descending thoracic aortic aneurysm. J Vasc Surg 1032; 51: 1026-32.

12) Erbel R, Alfonso F, Boileau C, Dirsch O, Eber B, et al. Diagnosis and management of aortic dissection. Eur Heart J 2001; 22: 1642-81.

13) Golledge J, Eagle KA. Acute aortic dissection. Lancet 2008; 372: 55-66.

14) Hagan PG, Nienaber CA, Isselbacher EM, Bruckman D, Karavite DJ, et al. The International Registry of Acute Aortic Dissection (IRAD): new insights into an old disease. JAMA 2000; 283: 897-903.

15) Dake MD, Kato N, Mitchell RS, Semba CP, Razavi $\mathrm{MK}$, et al. Endovascular stent-graft placement for the treatment of acute aortic dissection. N Engl J Med 1999; 340: 1546-52.

16) Leurs LJ, Bell R, Degrieck Y, Thomas S, Hobo R, et al. Endovascular treatment of thoracic aortic diseases: combined experience from the EUROSTAR and
United Kingdom Thoracic Endograft registries. J Vasc Surg 2004; 40: 670-9.

17) Buffolo E, da Fonseca JH, de Souza JA, Alves CM. Revolutionary treatment of aneurysms and dissections of descending aorta: the endovascular approach. Ann Thorac Surg 2002; 74: S1815-7.

18) Parker JD, Golledge J. Outcome of endovascular treatment of acute type B aortic dissection. Ann Thorac Surg 2008; 86: 1707-12.

19) Eggebrecht H, Nienaber CA, Neuhäuser M, Baumgart D, Kische S, et al. Endovascular stent-graft placement in aortic dissection: a meta-analysis. Eur Heart J 2006; 27: 489-98.

20) Nienaber CA, Rousseau H, Eggebrecht H, Kische S, Fattori R, et al. Randomized comparison of strategies for type B aortic dissection: the INvestigation of STEnt Grafts in Aortic Dissection (INSTEAD) trial. Circulation 2009; 120: 2519-28.

21) Eggebrecht H, Plicht B, Kahlert P, Erbel R. Intramural hematoma and penetrating ulcers: indications to endovascular treatment. Eur J Vasc Endovasc Surg 2009; 38: 659-65.

22) Sundt TM. Intramural hematoma and penetrating aortic ulcer. Curr Opin Cardiol 2007; 22: 504-9.

23) Stanson AW, Kazmier FJ, Hollier LH, Edwards WD, Pairolero PC, et al. Penetrating atherosclerotic ulcers of the thoracic aorta: natural history and clinicopathologic correlations. Ann Vasc Surg 1986; 1: 15-23.

24) PARMLEY LF, MATTINGLY TW, MANION WC, JAHNKE EJ Jr. Nonpenetrating traumatic injury of the aorta. Circulation 1958; 17: 1086-101.

25) Fernandez V, Mestres G, Maeso J, Dom Ãnguez JM, Aloy MC, et al. Endovascular treatment of traumatic thoracic aortic injuries: short- and medium-term Followup. Ann Vasc Surg 2010; 24: 160-6.

26) Go MR, Barbato JE, Dillavou ED, Gupta N, Rhee RY, et al. Thoracic endovascular aortic repair for traumatic aortic transection. J Vasc Surg 2007; 46: 928-33.

27) Xenos ES, Abedi NN, Davenport DL, Minion DJ, Hamdallah O, et al. Meta-analysis of endovascular vs open repair for traumatic descending thoracic aortic rupture. J Vasc Surg 2008; 48: 1343-51.

28) Jonker FH, Giacovelli JK, Muhs BE, Sosa JA, Indes JE. Trends and outcomes of endovascular and open treatment for traumatic thoracic aortic injury. J Vasc Surg 2010; 51: 565-71. 